\title{
Evaluation Formulas for Generalized Conditional Wiener Integrals with Drift on a Function Space
}

\author{
Dong Hyun Cho \\ Department of Mathematics, Kyonggi University, Suwon 443-760, Republic of Korea \\ Correspondence should be addressed to Dong Hyun Cho; j94385@kyonggi.ac.kr
}

Received 4 May 2013; Revised 23 September 2013; Accepted 23 September 2013

Academic Editor: Anna Kamińska

Copyright (C) 2013 Dong Hyun Cho. This is an open access article distributed under the Creative Commons Attribution License, which permits unrestricted use, distribution, and reproduction in any medium, provided the original work is properly cited.

\begin{abstract}
Let $C[0, t]$ denote a generalized Wiener space, the space of real-valued continuous functions on the interval $[0, t]$ and define a stochastic process $Y: C[0, t] \times[0, t] \rightarrow \mathbb{R}$ by $Y(x, s)=\int_{0}^{s} h(u) d x(u)+a(s)$ for $x \in C[0, t]$ and $s \in[0, t]$, where $h \in L_{2}[0, t]$ with $h \neq 0$ a.e. and $a$ is continuous on $[0, t]$. Let random vectors $Y_{n}: C[0, t] \rightarrow \mathbb{R}^{n}$ and $Y_{n+1}: C[0, t] \rightarrow \mathbb{R}^{n+1}$ be given by $Y_{n}(x)=\left(Y\left(x, t_{1}\right), \ldots, Y\left(x, t_{n}\right)\right)$ and $Y_{n+1}(x)=\left(Y\left(x, t_{1}\right), \ldots, Y\left(x, t_{n}\right), Y\left(x, t_{n+1}\right)\right)$, where $0<t_{1}<\cdots<t_{n}<t_{n+1}=t$ is a partition of $[0, t]$. In this paper we derive a translation theorem for a generalized Wiener integral and then prove that $Y$ is a generalized Brownian motion process with drift $a$. Furthermore, we derive two simple formulas for generalized conditional Wiener integrals of functions on $C[0, t]$ with the drift and the conditioning functions $Y_{n}$ and $Y_{n+1}$. As applications of these simple formulas, we evaluate the generalized conditional Wiener integrals of various functions on $C[0, t]$.
\end{abstract}

\section{Introduction}

Let $C_{0}[0, t]$ denote the Wiener space, the space of real-valued continuous functions $x$ on $[0, t]$ with $x(0)=0$. On the space, Yeh [1] introduced an inversion formula that a conditional expectation can be found by a Fourier-transform. But Yeh's inversion formula is very complicated in its application when the conditioning function is vector-valued. In [2], Park and Skoug derived a simple formula for conditional Wiener integrals on $C_{0}[0, t]$ with a vector-valued conditioning function $X: C_{0}[0, t] \rightarrow \mathbb{R}^{n}$ given by $X(x)=\left(x\left(t_{1}\right), \ldots, x\left(t_{n+1}\right)\right)$, where $0=t_{0}<t_{1}<\cdots<t_{n}<t_{n+1}=t$ is a partition of the interval $[0, t]$. In their simple formula, they expressed the conditional Wiener integral directly in terms of an ordinary Wiener integral. Using the simple formula in [2], Chang and Skoug [3] investigated the effect that drift has on the conditional Fourier-Feynman transform, the conditional convolution product, and various relationships that occur between them.

On the other hand, let $C[0, t]$ denote the space of realvalued continuous functions on the interval $[0, t]$. Im and Ryu [4] introduced a probability measure $w_{\varphi}$ on $C[0, t]$, where $\varphi$ is a probability measure on the Borel class of $\mathbb{R}$. When $\varphi=\delta_{0}$, the Dirac measure concentrated at $0, w_{\varphi}$ is exactly the Wiener measure on $C_{0}[0, t]$. On the space $C[0, t]$, the author $[5,6]$ derived two simple formulas for the conditional Wiener $w_{\varphi}$ integral of functions on $C[0, t]$ with the vector-valued conditioning functions $X_{n}: C[0, t] \rightarrow \mathbb{R}^{n+1}$ and $X_{n+1}: C[0, t] \rightarrow$ $\mathbb{R}^{n+2}$ given by $X_{n}(x)=\left(x\left(t_{0}\right), x\left(t_{1}\right), \ldots, x\left(t_{n}\right)\right)$ and $X_{n+1}(x)=\left(x\left(t_{0}\right), x\left(t_{1}\right), \ldots, x\left(t_{n}\right), x\left(t_{n+1}\right)\right)$ which generalize the Park and Skoug's formula in [2]. Using these formulas with the conditioning functions $X_{n}$ and $X_{n+1}$, he evaluated the conditional Wiener $w_{\varphi}$-integral of function of the form $F_{m}(x)=\int_{0}^{t}(x(s))^{m} d s$ for any positive integer $m$.

Let $h \in L_{2}[0, t]$ with $h \neq 0$ a.e. on $[0, t]$, and let $a$ be a continuous function on $[0, t]$. Define a stochastic process $Y$ : $C[0, t] \times[0, t] \rightarrow \mathbb{R}$ by $Y(x, s)=\int_{0}^{s} h(u) d x(u)+a(s)$ for $x \in C[0, t]$ and $s \in[0, t]$. Let $Y_{n}: C[0, t] \rightarrow \mathbb{R}^{n}$ and $Y_{n+1}:$ $C[0, t] \rightarrow \mathbb{R}^{n+1}$ be given by

$$
\begin{gathered}
Y_{n}(x)=\left(Y\left(x, t_{1}\right), \ldots, Y\left(x, t_{n}\right)\right), \\
Y_{n+1}(x)=\left(Y\left(x, t_{1}\right), \ldots, Y\left(x, t_{n}\right), Y\left(x, t_{n+1}\right)\right) .
\end{gathered}
$$


In this paper, we derive a translation theorem for a generalized Wiener $w_{\varphi}$-integral, and then prove that $Y$ is a generalized Brownian motion process with drift $a$ and variance $\int_{0}^{s}(h(u))^{2} d u$ for $s \in[0, t]$. Furthermore, we derive two simple formulas for generalized conditional Wiener integrals of functions on $C[0, t]$ with the drift and the conditioning functions $Y_{n}$ and $Y_{n+1}$. As applications of these simple formulas, we evaluate the generalized conditional Wiener integrals of functions of the forms

$$
\begin{gathered}
F_{m}(Y(x, \cdot)), \quad Y\left(x, s_{1}\right) Y\left(x, s_{2}\right), \\
\int_{L_{2}[0, t]} \exp \{i(v, Y(x, \cdot))\} d \sigma(v)
\end{gathered}
$$

for $x \in C[0, t]$, where $0<s_{1}<s_{2} \leq t$ and $\sigma$ is a complex Borel measure on $L_{2}[0, t]$.

\section{A Generalized Brownian Motion Process with Drift}

In this section, we introduce a generalized Brownian motion process with drift which generalizes the generalized Wiener space $C_{a, b}[0, T]$ as given in [7].

Let $\mathbb{C}$ and $\mathbb{C}_{+}$denote the sets of complex numbers and complex numbers with positive real parts, respectively. Let $\left(C[0, t], \mathscr{B}(C[0, t]), w_{\varphi}\right)$ be the analogue of Wiener space associated with a probability measure $\varphi$ on the Borel class of $\mathbb{R}$, where $\mathscr{B}(C[0, t])$ denotes the Borel class of $C[0, t][4]$. Let $\alpha \in \mathbb{R}$, and let $\varphi_{\alpha}$ be a measure on $(\mathbb{R}, \mathscr{B}(\mathbb{R}))$ such that $\varphi_{\alpha}(B)=\varphi(B+\alpha)$ for $B \in \mathscr{B}(\mathbb{R})$. For $v$ in $L_{2}[0, t]$ and $x$ in $C[0, t]$, let $(v, x)=\int_{0}^{t} v(s) d x(s)$ denote the Paley-WienerZygmund integral of $v$ according to $x$ [4]. Let $h \in L_{2}[0, t]$ with $h \neq 0$ a.e. on $[0, t]$, and let $a$ be a continuous function on $[0, t]$. Define stochastic processes $Z, Y: C[0, t] \times[0, t] \rightarrow \mathbb{R}$ by $Z(x, s)=\int_{0}^{s} h(u) d x(u)$ and $Y(x, s)=\int_{0}^{s} h(u) d x(u)+a(s)$ for $x \in C[0, t]$ and $s \in[0, t]$. Let $b(s)=\int_{0}^{s}(h(u))^{2} d u$ for $0 \leq s \leq t$.

By (3.1) in [4, Theorem 3.1], we have the following translation theorem for a generalized Wiener integral on the analogue of Wiener space.

Theorem 1. Let $g$ be of bounded variation on $[0, t]$, let $g_{0}(s)=$ $\int_{0}^{s} h(u) g(u) d u$ for $s \in[0, t]$, and let $F$ be a measurable function on $C[0, t]$. Then, $F\left(Y(x, \cdot)+g_{0}\right)$ is also measurable function of $x$ on $C[0, t]$ and

$$
\begin{aligned}
& \int_{C[0, t]} F(Y(x, \cdot)) d w_{\varphi}(x) \\
& \stackrel{*}{=} e^{-\|g\|^{2} / 2} \int_{C[0, t]} F\left(Y(x, \cdot)+g_{0}\right) \exp \{-(g, x)\} d w_{\varphi_{\alpha}}(x),
\end{aligned}
$$

where $\stackrel{*}{=}$ means that if one side exists then both sides exist, and they are equal.

Letting $g=\chi_{[0, s]} h$, we have the following corollary by Theorem 1 .
Corollary 2. Let $h$ be of bounded variation on $[0, t]$ and $F$ a measurable function on $C[0, t]$. Then, for $s \in[0, t] F(Y(x, \cdot)+$ $b(\min \{s, \cdot\}))$ is also measurable on $C[0, t]$ and

$$
\begin{aligned}
& \int_{C[0, t]} F(Y(x, \cdot)) d w_{\varphi}(x) \\
& \stackrel{*}{=} \exp \left\{-\frac{1}{2} b(s)\right\} \int_{C[0, t]} F(Y(x, \cdot)+b(\min \{s, \cdot\})) \\
& \quad \times \exp \{-Z(x, s)\} d w_{\varphi_{\alpha}}(x) .
\end{aligned}
$$

Letting $F \equiv 1$ in Theorem 1 and Corollary 2, we have the following corollary.

Corollary 3. Under the assumptions as given in Theorem 1 and Corollary 2

$$
\int_{C[0, t]} \exp \{-(g, x)\} d w_{\varphi_{\alpha}}(x)=\exp \left\{\frac{1}{2}\|g\|^{2}\right\} .
$$

In particular for $s \in[0, t]$

$$
\int_{C[0, t]} \exp \{-Z(x, s)\} d w_{\varphi_{\alpha}}(x)=\exp \left\{\frac{1}{2} b(s)\right\}
$$

Using the same method as used in the proof of Corollary 3.3 in [4], we have the following corollary.

Corollary 4. Under the assumptions as given in Theorem 1, one has for $\lambda \in \mathbb{C}$

$$
\int_{C[0, t]} \exp \{-\lambda(g, x)\} d w_{\varphi_{\alpha}}(x)=\exp \left\{\frac{\lambda^{2}}{2}\|g\|^{2}\right\}
$$

In particular for $s \in[0, t]$

$$
\int_{C[0, t]} \exp \{-\lambda Z(x, s)\} d w_{\varphi_{\alpha}}(x)=\exp \left\{\frac{\lambda^{2}}{2} b(s)\right\} .
$$

Letting $\lambda=-i \xi(\xi \in \mathbb{R})$ in the equations of Corollary 4 , we have the following corollary by Fourier transforms on $\left(C[0, t], w_{\varphi_{\alpha}}\right)$.

Corollary 5. Under the assumptions as given in Theorem 1, let $X(x)=(g, x)$. Then, $X$ is normally distributed with mean zero and variance $\|g\|^{2}$ on $\left(C[0, t], w_{\varphi_{\alpha}}\right)$ if $\|g\|>0$. Moreover, for $s \in$ $(0, t] Y(\cdot, s)$ is normally distributed with mean $a(s)$ and variance $b(s)$ on $\left(C[0, t], w_{\varphi_{\alpha}}\right)$. In particular, $Z(\cdot, s)$ is normally distributed on $\left(C[0, t], w_{\varphi_{\alpha}}\right)$ with mean 0 and variance $b(s)$.

Using the same method as used in the proof of Theorem 3.5 in [4], we have the following theorem.

Theorem 6. Let $\left\{g_{1}, \ldots, g_{m}\right\}$ be an orthonormal subset of $L_{2}[0, t]$ such that each $g_{j}$ is of bounded variation. For $j=$ $1, \ldots, m$, let $X_{j}(x)=\left(g_{j}, x\right)$. Then $, X_{1}, \ldots, X_{m}$ are independent, 
and each $X_{j}$ has the standard normal distribution. Moreover if, $f: \mathbb{R}^{m} \rightarrow \mathbb{C}$ is Borel measurable, then

$$
\begin{aligned}
& \int_{C[0, t]} f\left(X_{1}(x), \ldots, X_{m}(x)\right) d w_{\varphi_{\alpha}}(x) \\
& \stackrel{*}{=}\left(\frac{1}{2 \pi}\right)^{m / 2} \int_{\mathbb{R}^{m}} f\left(u_{1}, u_{2}, \ldots, u_{m}\right) \\
& \quad \times \exp \left\{-\frac{1}{2} \sum_{j=1}^{m} u_{j}^{2}\right\} d\left(u_{1}, u_{2}, \ldots, u_{m}\right) .
\end{aligned}
$$

Theorem 7. Let $h$ be of bounded variation on $[0, t]$, and let $0=s_{0}<s_{1}<\cdots<s_{m} \leq t$. Then, the random vector $\left(Y\left(\cdot, s_{1}\right), \ldots, Y\left(\cdot, s_{m}\right)\right)$ has a joint density function $W_{m}$ with respect to $w_{\varphi_{\alpha}}$ given by

$$
\begin{aligned}
W_{m} & \left(s_{1}, \ldots, s_{m} ; u_{1}, \ldots, u_{m}\right) \\
= & \prod_{j=1}^{m}\left[\frac{1}{2 \pi\left(b\left(s_{j}\right)-b\left(s_{j-1}\right)\right)}\right]^{1 / 2} \\
& \quad \times \exp \left\{-\sum_{j=1}^{m} \frac{\left(u_{j}-a\left(s_{j}\right)-\left(u_{j-1}-a\left(s_{j-1}\right)\right)\right)^{2}}{2\left(b\left(s_{j}\right)-b\left(s_{j-1}\right)\right)}\right\},
\end{aligned}
$$

where $u_{0}=0, a(0)=0$, and $\left(u_{1}, \ldots, u_{m}\right) \in \mathbb{R}^{m}$.

Proof. For $j=1, \ldots, m$, let $h_{j}=\chi_{\left[s_{j-1}, s_{j}\right]} h$, where $\chi_{\left[s_{j-1}, s_{j}\right]}$ denotes the indicator function on $\left[s_{j-1}, s_{j}\right]$. Let $g_{j}=$ $\left(1 / \sqrt{b\left(s_{j}\right)-b\left(s_{j-1}\right)}\right) h_{j}$. Then, $g_{1}, \ldots, g_{m}$ are orthonormal, and $Y\left(\cdot, s_{j}\right)=\sum_{l=1}^{j}\left(h_{l}, \cdot\right)+a\left(s_{j}\right)=\sum_{l=1}^{j} \sqrt{b\left(s_{l}\right)-b\left(s_{l-1}\right)}\left(g_{l}, \cdot\right)+$ $a\left(s_{j}\right)$. Let $B$ be a Borel subset of $\mathbb{R}^{m}$. By Theorem 6 ,

$$
\begin{gathered}
w_{\varphi_{\alpha}}\left(\left(Y\left(\cdot, s_{1}\right), \ldots, Y\left(\cdot, s_{m}\right)\right) \in B\right) \\
=\left(\frac{1}{2 \pi}\right)^{m / 2} \int_{\mathbb{R}^{m}} \chi_{B}\left(\sqrt{b\left(s_{1}\right)-b\left(s_{0}\right)} w_{1}+a\left(s_{1}\right), \ldots,\right. \\
\left.\sum_{l=1}^{m} \sqrt{b\left(s_{l}\right)-b\left(s_{l-1}\right)} w_{l}+a\left(s_{m}\right)\right) \\
\times \exp \left\{-\frac{1}{2} \sum_{j=1}^{m} w_{j}^{2}\right\} d\left(w_{1}, \ldots, w_{m}\right) .
\end{gathered}
$$

For $j=1, \ldots, m$, let $u_{j}=\sum_{l=1}^{j} \sqrt{b\left(s_{l}\right)-b\left(s_{l-1}\right)} w_{l}+a\left(s_{j}\right)$. Then, by the change of variable theorem,

$$
\begin{aligned}
w_{\varphi_{\alpha}} & \left(\left(Y\left(\cdot, s_{1}\right), \ldots, Y\left(\cdot, s_{m}\right)\right) \in B\right) \\
\quad & \int_{B} w_{m}\left(s_{1}, \ldots, s_{m} ; u_{1}, \ldots, u_{m}\right) d\left(u_{1}, \ldots, u_{m}\right)
\end{aligned}
$$

which completes the proof.

Remark 8. The condition $a(0)=0$ in Theorem 7 does not mean that the mean function $a$ is continuous on $[0, t]$ with $a(0)=0$. In fact, it is only needed to express formally the exponential function $\exp \left\{-\left(u_{1}-a\left(s_{1}\right)\right)^{2} /\left(2 b\left(s_{1}\right)\right)\right\}$ in $W_{m}$.

By Theorem 7 and the change of variable theorem, we have the following corollary.

Corollary 9. $Y$ is a Gaussian process with respect to $w_{\varphi_{\alpha}}$, and its covariance function is given by

$$
\begin{aligned}
& E\left[\left(Y\left(\cdot, s_{1}\right)-a\left(s_{1}\right)\right)\left(Y\left(\cdot, s_{2}\right)-a\left(s_{2}\right)\right)\right] \\
& \quad=b\left(\min \left\{s_{1}, s_{2}\right\}\right) \quad \text { for } s_{1}, s_{2} \in[0, t],
\end{aligned}
$$

so that

$$
E\left[Y\left(\cdot, s_{1}\right) Y\left(\cdot, s_{2}\right)\right]=b\left(\min \left\{s_{1}, s_{2}\right\}\right)+a\left(s_{1}\right) a\left(s_{2}\right) .
$$

Using Fourier transforms on $\left(C[0, t], w_{\varphi_{\alpha}}\right)$, we have the following corollary by Theorem 7 .

Corollary 10. Let $0 \leq s_{1}<s_{2} \leq t$. Then, $Y\left(\cdot, s_{2}\right)-Y\left(\cdot, s_{1}\right)$ is normally distributed on $\left(C[0, t], w_{\varphi_{\alpha}}\right)$ with mean $a\left(s_{2}\right)-a\left(s_{1}\right)$ and variance $b\left(s_{2}\right)-b\left(s_{1}\right)$.

By Corollary 10, we can prove the following corollary.

Corollary 11. Let $0 \leq s_{1}<s_{2} \leq s_{3}<s_{4} \leq t$. Then $Y\left(\cdot, s_{2}\right)-$ $Y\left(\cdot, s_{1}\right)$ and $Y\left(\cdot, s_{4}\right)-Y\left(\cdot, s_{3}\right)$ are independent on $\left(C[0, t], w_{\varphi_{\alpha}}\right)$.

Theorem 12. $Y$ is a generalized Brownian motion process on $\left(C[0, t], w_{\varphi_{\alpha}}\right)$ determined by the mean function $a$ and variance function $b$.

Remark 13. (1) A generalized Brownian motion process determined by the mean function $a$ and variance function $b$ is a Gaussian process whose mean and covariance functions are given by $a$ and $b\left(\min \left\{s_{1}, s_{2}\right\}\right)-b(0)$, respectively, for $s_{1}, s_{2} \in$ $[0, t][1]$.

(2) In [8], the author proved that $Y-a$ is a generalized Brownian motion process using Theorem 6. Comparing with [8], we proved the results in this paper using only the translation theorem [4, Theorem 3.1] which is another approach to prove $Y$ being a generalized Brownian motion process.

\section{Simple Formulas for Generalized Conditional Wiener Integrals}

In this section, we derive two simple formulas for generalized conditional Wiener integrals on $\left(C[0, t], w_{\varphi_{\alpha}}\right)$. For this purpose, we start with this section defining a conditional $w_{\varphi_{\alpha}}{ }^{-}$ integral.

Let $F: C[0, t] \rightarrow \mathbb{C}$ be integrable on $w_{\varphi_{\alpha}}$ and $X$ a random vector on $C[0, t]$ assuming that the value space of $X$ is a normed space equipped with the Borel $\sigma$-algebra. Then, we have the conditional expectation $E[F \mid X]$ of $F$ given $X$ from a well-known probability theory. Furthermore, there exists a $P_{X}$-integrable $\mathbb{C}$-valued function $\psi$ on the value space of $X$ such that $E[F \mid X](x)=(\psi \circ X)(x)$ for $w_{\varphi_{\alpha}}$ a.e. $x \in C[0, t]$, where $P_{X}$ is the probability distribution of $X$. The function $\psi$ is called the conditional $w_{\varphi_{\alpha}}$-integral of $F$ given $X$, and it is 
also denoted by $E[F \mid X]$. Let $0=t_{0}<t_{1}<\cdots<t_{n}<t_{n+1}=t$ be a partition of $[0, t]$. For $j=1, \ldots, n+1$, let $\alpha_{j}(s)=$ $\left(b\left(t_{j}\right)-b(s)\right) /\left(b\left(t_{j}\right)-b\left(t_{j-1}\right)\right), \beta_{j}(s)=\left(b(s)-b\left(t_{j-1}\right)\right) /\left(b\left(t_{j}\right)-\right.$ $\left.b\left(t_{j-1}\right)\right)$ and $\gamma_{j}(s)=\alpha_{j}(s) \beta_{j}(s)\left(b\left(t_{j}\right)-b\left(t_{j-1}\right)\right)$, where $s \in$ $\left[t_{j-1}, t_{j}\right]$. Define random vectors $Y_{n}: C[0, t] \rightarrow \mathbb{R}^{n}$ and $Y_{n+1}: C[0, t] \rightarrow \mathbb{R}^{n+1}$ by $Y_{n}(x)=\left(Y\left(x, t_{1}\right), \ldots, Y\left(x, t_{n}\right)\right)$ and $Y_{n+1}(x)=\left(Y\left(x, t_{1}\right), \ldots, Y\left(x, t_{n}\right), Y\left(x, t_{n+1}\right)\right)$ for $x \in C[0, t]$. For any $x$ in $C[0, t]$, define a polygonal function $[Y(x, \cdot)]_{a, b}$ of $Y(x, \cdot)$ by

$$
\begin{aligned}
& {[Y(x, \cdot)]_{a, b}(s)} \\
& \quad=\sum_{j=1}^{n+1} \chi_{\left(t_{j-1}, t_{j}\right]}(s)\left(\alpha_{j}(s) Y\left(x, t_{j-1}\right)+\beta_{j}(s) Y\left(x, t_{j}\right)\right) \\
& \quad+\chi_{\{0\}}(s) a(0)
\end{aligned}
$$

for $s \in[0, t]$, where $\chi_{\left(t_{j-1}, t_{j}\right]}$ and $\chi_{\{0\}}$ denote indicator functions. For $x \in C[0, t]$ and $\vec{\xi}_{n+1}=\left(\xi_{1}, \ldots, \xi_{n}, \xi_{n+1}\right) \in \mathbb{R}^{n+1}$, define polygonal functions $[Z(x, \cdot)]_{b}$ and $\left[\vec{\xi}_{n+1}\right]_{b}$ of $Z(x, \cdot)$ and $\vec{\xi}_{n+1}$ by (15), where $Y\left(x, t_{j}\right)$ is replaced by $Z\left(x, t_{j}\right)$ and $\xi_{j}$, respectively, for $j=1, \ldots, n+1\left(\xi_{0}=0\right.$ and $a(0)=0$ formally). Moreover, define a polygonal function $[a]_{b}$ of $a$ by (15), where $Y\left(x, t_{j}\right)$ is replaced by $a\left(t_{j}\right)$. For $\vec{\xi}_{n}=\left(\xi_{1}, \ldots, \xi_{n}\right) \in \mathbb{R}^{n}$, the symbol $\left[\vec{\xi}_{n}\right]_{b}$ is understood as $\chi_{\left[0, t_{n}\right]}\left[\vec{\xi}_{n+1}\right]_{b}$ on $[0, t]$.

By Theorems 2.4 and 2.9 in [8], we can easily prove the following theorem.

Theorem 14. Let $t_{j-1}<s<t_{j}$ for some $j \in\{1, \ldots, n+1\}$. Then, $Y(\cdot, s)-[Y(\cdot, \cdot)]_{a, b}(s)$ is normally distributed on $\left(C[0, t], w_{\varphi_{\alpha}}\right)$ with mean $a(s)-[a]_{b}(s)$ and variance $\gamma_{j}(s)$.

Theorem 15. The process $\left\{Y(\cdot, s)-[Y(\cdot, \cdot)]_{a, b}(s): 0 \leq s \leq t\right\}$ and $Y_{n+1}$ are stochastically independent on $\left(C[0, t], w_{\varphi_{\alpha}}\right)$.

Proof. Let $t_{l-1}<s<t_{l}$ for some $l \in\{1, \ldots, n+1\}$. Then, for $j=1, \ldots, n+1$,

$$
\begin{gathered}
E\left[\left(Y(\cdot, s)-[Y(\cdot, \cdot)]_{a, b}(s)\right) Y\left(\cdot, t_{j}\right)\right] \\
=E\left[\left(Z(\cdot, s)-[Z(\cdot, \cdot)]_{b}(s)+a(s)\right.\right. \\
\left.\left.\quad-[a]_{b}(s)\right)\left(Z\left(\cdot, t_{j}\right)+a\left(t_{j}\right)\right)\right] \\
=E\left[Z(\cdot, s)-[Z(\cdot, \cdot)]_{b}(s)\right] E\left[Z\left(\cdot, t_{j}\right)\right] \\
+\left(a(s)-[a]_{b}(s)\right) a\left(t_{j}\right)
\end{gathered}
$$

by Corollary 2.4 and Theorems 2.9 and 2.10 in [8]. By Corollary 5 and Theorem 14 we have

$$
\begin{aligned}
E[ & \left.\left(Y(\cdot, s)-[Y(\cdot, \cdot)]_{a, b}(s)\right) Y\left(\cdot, t_{j}\right)\right] \\
& =\left(a(s)-[a]_{b}(s)\right) a\left(t_{j}\right) \\
& =E\left[Y(\cdot, s)-[Y(\cdot, \cdot)]_{a, b}(s)\right] E\left[Y\left(\cdot, t_{j}\right)\right] .
\end{aligned}
$$

Since $Y(\cdot, s)-[Y(\cdot, \cdot)]_{a, b}(s)$ and $Y\left(\cdot, t_{j}\right)$ are normally distributed on $w_{\varphi_{\alpha}}$, they are independent. The proofs of remainder cases follow easily.

Theorem 16. The processes $\left\{Y(\cdot, s)-[Y(\cdot, \cdot)]_{a, b}(s): t_{j-1} \leq s \leq\right.$ $\left.t_{j}\right\}$, where $j=1, \ldots, n+1$, are stochastically independent on $\left(C[0, t], w_{\varphi_{\alpha}}\right)$.

Proof. Let $s_{1} \in\left[t_{l-1}, t_{l}\right]$ and $s_{2} \in\left[t_{j-1}, t_{j}\right]$ with $l<j$. If $s_{1} \in$ $\left\{0, t_{1}, \ldots, t_{n+1}\right\}$ or $s_{2} \in\left\{0, t_{1}, \ldots, t_{n+1}\right\}$, we can prove easily the independence of $Y\left(\cdot, s_{1}\right)-[Y(\cdot, \cdot)]_{a, b}\left(s_{1}\right)$ and $Y\left(\cdot, s_{2}\right)-$ $[Y(\cdot, \cdot)]_{a, b}\left(s_{2}\right)$. Suppose that $t_{l-1}<s_{1}<t_{l} \leq t_{j-1}<s_{2}<t_{j}$. By Theorems 3.1 and 2.11 in [8], we have

$$
\begin{aligned}
E\left[\left(Y\left(\cdot, s_{1}\right)-[Y(\cdot, \cdot)]_{a, b}\left(s_{1}\right)\right)\left(Y\left(\cdot, s_{2}\right)-[Y(\cdot, \cdot)]_{a, b}\left(s_{2}\right)\right)\right] \\
=E\left[\left(Z\left(\cdot, s_{1}\right)-[Z(\cdot, \cdot)]_{b}\left(s_{1}\right)+a\left(s_{1}\right)-[a]_{b}\left(s_{1}\right)\right)\right. \\
\left.\quad \times\left(Z\left(\cdot, s_{2}\right)-[Z(\cdot, \cdot)]_{b}\left(s_{2}\right)+a\left(s_{2}\right)-[a]_{b}\left(s_{2}\right)\right)\right] \\
=E\left[Z\left(\cdot, s_{1}\right)-[Z(\cdot, \cdot)]_{b}\left(s_{1}\right)\right] E\left[Z\left(\cdot, s_{2}\right)-[Z(\cdot, \cdot)]_{b}\left(s_{2}\right)\right] \\
+\left(a\left(s_{1}\right)-[a]_{b}\left(s_{1}\right)\right)\left(a\left(s_{2}\right)-[a]_{b}\left(s_{2}\right)\right) \\
=E\left[Y\left(\cdot, s_{1}\right)-[Y(\cdot, \cdot)]_{a, b}\left(s_{1}\right)\right] E\left[Y\left(\cdot, s_{2}\right)-[Y(\cdot, \cdot)]_{a, b}\left(s_{2}\right)\right]
\end{aligned}
$$

which completes the proof.

Applying the same method as used in the proof of Theorem 2 in [2, page 383] with Problem 4 of [9, page 216], we have the following theorem by Theorem 15 .

Theorem 17. Let $F: C[0, t] \rightarrow \mathbb{C}$ be a function, and let $F(Y(x, \cdot))$ be $w_{\varphi_{\alpha}}$-integrable over the variable $x$. Then, for a Borel subset $B$ of $\mathbb{R}^{n}$

$$
\begin{aligned}
& \int_{Y_{n+1}^{-1}(B)} F(Y(x, \cdot)) d w_{\varphi_{\alpha}}(x) \\
& \quad=\int_{B} E\left[F\left(Y(x, \cdot)-[Y(x, \cdot)]_{a, b}+\left[\vec{\xi}_{n+1}\right]_{b}\right)\right] d P_{Y_{n+1}}\left(\vec{\xi}_{n+1}\right),
\end{aligned}
$$

where the expectation is taken over $w_{\varphi_{\alpha}}$ and $P_{Y_{n+1}}$ is the probability distribution of $Y_{n+1}$ on $\left(\mathbb{R}^{n+1}, \mathscr{B}\left(\mathbb{R}^{n+1}\right)\right)$. Moreover, for $P_{Y_{n+1}}$ a.e. $\vec{\xi}_{n+1} \in \mathbb{R}^{n+1}$ (hence for a.e. $\vec{\xi}_{n+1} \in \mathbb{R}^{n+1}$ )

$$
\begin{aligned}
E & {\left[F(Y(x, \cdot)) \mid Y_{n+1}\right]\left(\vec{\xi}_{n+1}\right) } \\
& =E\left[F\left(Y(x, \cdot)-[Y(x, \cdot)]_{a, b}+\left[\vec{\xi}_{n+1}\right]_{b}\right)\right] .
\end{aligned}
$$

Using the same method as used in the proof of Theorem 2.5 in [6], we can prove the following theorem.

Theorem 18. Let $F: C[0, t] \rightarrow \mathbb{C}$ be a function and $F(Y(x, \cdot)) w_{\varphi_{\alpha}}$-integrable over the variable $x$. Moreover, let $P_{Y_{n}}$ 
be a probability distribution of $Y_{n}$ on $\left(\mathbb{R}^{n}, \mathscr{B}\left(\mathbb{R}^{n}\right)\right)$. Then, for $P_{Y_{n}}$ a.e. $\vec{\xi}_{n}=\left(\xi_{1}, \ldots, \xi_{n}\right) \in \mathbb{R}^{n}$ (hence for a.e. $\vec{\xi}_{n} \in \mathbb{R}^{n}$ )

$$
\begin{aligned}
& E\left[F \mid Y_{n}\right]\left(\vec{\xi}_{n}\right) \\
& =\left[\frac{1}{2 \pi\left(b(t)-b\left(t_{n}\right)\right)}\right]^{1 / 2} \\
& \quad \times \int_{\mathbb{R}} E\left[F\left(Y(x, \cdot)-[Y(x, \cdot)]_{a, b}+\left[\vec{\xi}_{n+1}\right]_{b}\right)\right] \\
& \quad \times \exp \left\{-\frac{\left(\xi_{n+1}-a(t)-\left(\xi_{n}-a\left(t_{n}\right)\right)\right)^{2}}{2\left(b(t)-b\left(t_{n}\right)\right)}\right\} d \xi_{n+1},
\end{aligned}
$$

where $\vec{\xi}_{n+1}=\left(\xi_{1}, \ldots, \xi_{n}, \xi_{n+1}\right)$ and the expectation is taken over $w_{\varphi_{\alpha}}$.

Note that the conditioning functions $Y_{n+1}$ and $Y_{n}$ describe the positions of paths at the times $t_{1}, \ldots, t_{n}, t_{n+1}=t$ (the present time). $Y_{n+1}$ contains the present position $Y(x, t)$ of the path $Y(x, \cdot)$ for $x \in C[0, t]$, while $Y_{n}$ does not. Moreover if we let $h=1$ a.e., $\alpha=0$, and $\varphi=\delta_{0}$, the Dirac measure concentrated at 0 , then we can obtain Theorems 1 and 2 in [10] by the translation theorem (Theorem 1). If $a(0)=0, h=\sqrt{b^{\prime}}$, $\varphi_{\alpha}=\delta_{0}$, and $t=T$, then we can obtain the space $C_{a, b}[0, T]$ in [7] by Theorem 12. Furthermore, if $Y$ is replaced by the generalized Brownian motion process $x(s)$ on $C_{a, b}[0, T] \times$ $[0, T]$, and we let $\varphi_{\alpha}=\delta_{0}$, then we can also obtain Theorem 3.4 in [7] by Theorem 17. If we let $a \equiv 0$ and $\varphi_{\alpha}=\delta_{0}$, then we can obtain Theorem 3 in [11] by Theorem 17. If we let $a \equiv 0$, then we can obtain Theorem 2.12 in [8] by Theorem 17. If we let $n=0$ and $\varphi_{\alpha}=\delta_{0}$, then we can obtain Remark 2.2 in [3] by Theorem 17. Finally, if we let $h=1$ a.e., $\varphi_{\alpha}=\delta_{0}$ and $a \equiv 0$, then we can obtain Theorem 2 in [2] by Theorem 17 which is among the first result expressing the conditional Wiener integrals of functions on $C_{0}[0, t]$ as ordinary Wiener integrals.

Remark 19. Note that Theorems 17 and 18 are not generalizations of Theorem 2.9 in [5] and Theorem 2.5 in [6]. In Theorem 2.9 of [5] and Theorem 2.5 of [6], the conditioning functions have initial distributions $\varphi$, while $Y_{n+1}$ and $Y_{n}$ in Theorems 17 and 18 have no initial distributions.

\section{Evaluation Formulas Using the Simple Formulas}

In this section, we derive evaluation formulas for the generalized conditional Wiener integrals of various functions which are of interest in Feynman integration theories and quantum mechanics. For a function $F: C[0, t] \rightarrow \mathbb{C}$ let $F_{Y}(x)=$ $F(Y(x, \cdot))$ for $x \in C[0, t]$.

Lemma 20. Let $F_{m}(x)=\int_{0}^{t}(x(s))^{m} d s(m \in \mathbb{N})$ for $x \in C[0, t]$. Then, $\left(F_{m}\right)_{Y}$ is $w_{\varphi_{\alpha}}$-integrable and

$$
E\left[\left(F_{m}\right)_{Y}\right]=\sum_{l=0}^{[m / 2]} \frac{m !}{2^{l} l !(m-2 l) !} \int_{0}^{t}(a(s))^{m-2 l}(b(s))^{l} d s,
$$

where [.] denotes the greatest integer function.
Proof. By Corollary 5, the change of variable theorem and the binomial expansion,

$$
\begin{aligned}
& \int_{0}^{t} \int_{C[0, t]}|Y(x, s)|^{m} d w_{\varphi_{\alpha}}(x) d s \\
& \leq\left(\frac{1}{\pi}\right)^{1 / 2} \sum_{l=0}^{m} 2^{l / 2} \Gamma\left(\frac{l+1}{2}\right)\left(\begin{array}{c}
m \\
l
\end{array}\right) \int_{(0, t]}|a(s)|^{m-l}(b(s))^{l / 2} d s,
\end{aligned}
$$

where $\Gamma$ denote the gamma function, so that $\left(F_{m}\right)_{Y}$ is integrable over $\left(C[0, t], w_{\varphi_{\alpha}}\right)$. Furthermore, we have

$$
\begin{aligned}
\int_{C[0, t]} & \int_{0}^{t}(Y(x, s))^{m} d s d w_{\varphi_{\alpha}}(x) \\
= & 2 \sum_{l=0}^{[m / 2]}\left(\begin{array}{l}
m \\
2 l
\end{array}\right) \int_{(0, t]}(a(s))^{m-2 l}\left(\frac{1}{2 \pi b(s)}\right)^{1 / 2} \\
& \times \int_{0}^{\infty} u^{2 l} \exp \left\{-\frac{u^{2}}{2 b(s)}\right\} d u d s \\
= & \sum_{l=0}^{[m / 2]} \frac{m !}{2^{l} l !(m-2 l) !} \int_{0}^{t}(a(s))^{m-2 l}(b(s))^{l} d s
\end{aligned}
$$

which completes the proof.

Theorem 21. Let the assumptions be as given in Lemma 20. Then, for a.e. $\vec{\xi}_{n+1} \in \mathbb{R}^{n+1}$ (hence, $P_{Y_{n+1}}$ a.e. $\vec{\xi}_{n+1} \in \mathbb{R}^{n+1}$ )

$$
\begin{aligned}
E & {\left[\left(F_{m}\right)_{Y} \mid Y_{n+1}\right]\left(\vec{\xi}_{n+1}\right) } \\
= & \sum_{j=1}^{n+1} \sum_{l=0}^{m / 2]} \frac{m !}{2^{l} l !(m-2 l) !} \\
& \times \int_{t_{j-1}}^{t_{j}}\left(a(s)-[a]_{b}(s)+\left[\vec{\xi}_{n+1}\right]_{b}(s)\right)^{m-2 l}\left(\gamma_{j}(s)\right)^{l} d s,
\end{aligned}
$$

where the expectation is taken over $w_{\varphi_{\alpha}}$.

Proof. For $P_{Y_{n+1}}$ a.e. $\vec{\xi}_{n+1} \in \mathbb{R}^{n+1}$, we have by Theorems 14 and 17

$$
\begin{aligned}
E[( & \left.\left.F_{m}\right)_{Y} \mid Y_{n+1}\right]\left(\vec{\xi}_{n+1}\right) \\
= & \sum_{j=1}^{n+1} \int_{\left(t_{j-1}, t_{j}\right)}\left(\frac{1}{2 \pi \gamma_{j}(s)}\right)^{1 / 2} \\
& \times \int_{\mathbb{R}}\left(u+a(s)-[a]_{b}(s)+\left[\vec{\xi}_{n+1}\right]_{b}(s)\right)^{m} \\
& \times \exp \left\{-\frac{u^{2}}{2 \gamma_{j}(s)}\right\} d u d s \\
= & \sum_{j=1}^{n+1} \sum_{l=0}^{m / 2]} \frac{m !}{2^{l} l !(m-2 l) !} \\
& \times \int_{t_{j-1}}^{t_{j}}\left(a(s)-[a]_{b}(s)+\left[\vec{\xi}_{n+1}\right]_{b}(s)\right)^{m-2 l}\left(\gamma_{j}(s)\right)^{l} d s
\end{aligned}
$$

by the same method as used in the proof of Lemma 20. 
Theorem 22. Let the assumptions be as given in Theorem 21, and for $\vec{\xi}_{n}=\left(\xi_{1}, \ldots, \xi_{n}\right) \in \mathbb{R}^{n}$ let

$$
\begin{aligned}
\Xi\left(\vec{\xi}_{n}\right)= & \sum_{j=1}^{n} \sum_{l=0}^{[m / 2]} \frac{m !}{2^{l} l !(m-2 l) !} \\
& \times \int_{t_{j-1}}^{t_{j}}\left(a(s)-[a]_{b}(s)+\left[\vec{\xi}_{n}\right]_{b}(s)\right)^{m-2 l}\left(\gamma_{j}(s)\right)^{l} d s .
\end{aligned}
$$

Then, for a.e. $\vec{\xi}_{n} \in \mathbb{R}^{n}$ (hence $P_{Y_{n}}$ a.e. $\vec{\xi}_{n} \in \mathbb{R}^{n}$ )

$$
\begin{aligned}
E & {\left[\left(F_{m}\right)_{Y} \mid Y_{n}\right]\left(\vec{\xi}_{n}\right) } \\
= & \Xi\left(\vec{\xi}_{n}\right)+m ! \\
& \times \sum_{l=0}^{[m / 2]} \sum_{k=0}^{m-2 l} \sum_{p=0}^{k} \sum_{q=0}^{[p / 2]} \frac{\xi_{n}^{k-p}\left(a(t)-a\left(t_{n}\right)\right)^{p-2 q}\left(b(t)-b\left(t_{n}\right)\right)^{q}}{2^{l+q l ! q !}(k-p) !(p-2 q) !(m-2 l-k) !} \\
& \times \int_{t_{n}}^{t}\left(\gamma_{n+1}(s)\right)^{l}\left(\beta_{n+1}(s)\right)^{p}\left(a(s)-[a]_{b}(s)\right)^{m-2 l-k} d s,
\end{aligned}
$$

where the expectation is taken over $w_{\varphi_{\alpha}}$.

Proof. For $\vec{\xi}_{n}=\left(\xi_{1}, \ldots, \xi_{n}\right) \in \mathbb{R}^{n}$, let $\vec{\xi}_{n+1}=\left(\xi_{1}, \ldots, \xi_{n}, \xi_{n+1}\right)$. By the binomial expansion, we have for $t_{n} \leq s \leq t$

$$
\begin{aligned}
&\left(a(s)-[a]_{b}(s)+\left[\vec{\xi}_{n+1}\right]_{b}(s)\right)^{m-2 l} \\
&=\sum_{k=0}^{m-2 l} \sum_{p=0}^{k} \frac{\xi_{n}^{k-p}(m-2 l) !}{p !(k-p) !(m-2 l-k) !}\left(\beta_{n+1}(s)\right)^{p} \\
& \times\left(a(s)-[a]_{b}(s)\right)^{m-2 l-k}\left(\xi_{n+1}-\xi_{n}\right)^{p},
\end{aligned}
$$

so that by Theorems 18 and 21 , we have for a.e. $\vec{\xi}_{n}=$ $\left(\xi_{1}, \cdots, \xi_{n}\right) \in \mathbb{R}^{n}$

$$
\begin{aligned}
& E\left[\left(F_{m}\right)_{Y} \mid Y_{n}\right]\left(\vec{\xi}_{n}\right) \\
& =\Xi\left(\vec{\xi}_{n}\right) \\
& +\sum_{l=0}^{[m / 2]} \sum_{k=0}^{m-2 l} \sum_{p=0}^{k} \sum_{q=0}^{[p / 2]} \frac{m ! \xi_{n}^{k-p}\left(a(t)-a\left(t_{n}\right)\right)^{p-2 q}}{2^{l-1} l !(2 q) !(k-p) !(p-2 q) !(m-2 l-k) !} \\
& \quad \times \int_{t_{n}}^{t}\left(\gamma_{n+1}(s)\right)^{l}\left(\beta_{n+1}(s)\right)^{p} \\
& \quad \times\left(a(s)-[a]_{b}(s)\right)^{m-2 l-k}\left[\frac{1}{2 \pi\left(b(t)-b\left(t_{n}\right)\right)}\right]^{1 / 2} \\
& \quad \times \int_{0}^{\infty} u^{2 q} \exp \left\{-\frac{u^{2}}{2\left(b(t)-b\left(t_{n}\right)\right)}\right\} d u d s
\end{aligned}
$$

by the change of variable theorem. Using the same method as used in the proof of Lemma 20, we have the desired result.
From now on, we assume that every expectation is taken over $w_{\varphi_{\alpha}}$ unless otherwise specified. By Corollary 9, Theorems 14 and 17, and Theorem 3.4 in [8], we have the following theorem.

Theorem 23. Let $0<s_{1}<s_{2} \leq t, s_{1} \in\left[t_{l-1}-t_{l}\right]$ and $s_{2} \in$ $\left[t_{j-1}-t_{j}\right]$. For $x \in C[0, t]$, let $G(x)=x\left(s_{1}\right) x\left(s_{2}\right)$.

(1) If $l \neq j$, then for a.e. $\vec{\xi}_{n+1} \in \mathbb{R}^{n+1}$ (hence, $P_{Y_{n+1}}$ a.e. $\left.\vec{\xi}_{n+1} \in \mathbb{R}^{n+1}\right)$

$$
\begin{aligned}
E\left[G_{Y} \mid Y_{n+1}\right]\left(\vec{\xi}_{n+1}\right) \\
=\left(a\left(s_{1}\right)-[a]_{b}\left(s_{1}\right)+\left[\vec{\xi}_{n+1}\right]_{b}\left(s_{1}\right)\right) \\
\quad \times\left(a\left(s_{2}\right)-[a]_{b}\left(s_{2}\right)+\left[\vec{\xi}_{n+1}\right]_{b}\left(s_{2}\right)\right) .
\end{aligned}
$$

(2) If $l=j$, then for a.e. $\vec{\xi}_{n+1} \in \mathbb{R}^{n+1}$ (hence, $P_{Y_{n+1}}$ a.e. $\vec{\xi}_{n+1} \in \mathbb{R}^{n+1}$ )

$$
\begin{aligned}
E\left[G_{Y} \mid Y_{n+1}\right]\left(\vec{\xi}_{n+1}\right) \\
=\alpha_{j}\left(s_{2}\right)\left(b\left(s_{1}\right)-b\left(t_{j-1}\right)\right) \\
+\left(a\left(s_{1}\right)-[a]_{b}\left(s_{1}\right)+\left[\vec{\xi}_{n+1}\right]_{b}\left(s_{1}\right)\right) \\
\quad \times\left(a\left(s_{2}\right)-[a]_{b}\left(s_{2}\right)+\left[\vec{\xi}_{n+1}\right]_{b}\left(s_{2}\right)\right) .
\end{aligned}
$$

Theorem 24. Let the assumptions be as given in Theorem 23.

(1) If $l \leq n, j \leq n$ and $l \neq j$, then for a.e. $\vec{\xi}_{n} \in \mathbb{R}^{n}$ (hence, $P_{Y_{n}}$ a.e. $\vec{\xi}_{n} \in \mathbb{R}^{n}$ )

$$
\begin{aligned}
E\left[G_{Y} \mid Y_{n}\right]\left(\vec{\xi}_{n}\right)= & \left(a\left(s_{1}\right)-[a]_{b}\left(s_{1}\right)+\left[\vec{\xi}_{n}\right]_{b}\left(s_{1}\right)\right) \\
& \times\left(a\left(s_{2}\right)-[a]_{b}\left(s_{2}\right)+\left[\vec{\xi}_{n}\right]_{b}\left(s_{2}\right)\right) .
\end{aligned}
$$

(2) If $l \leq n, j \leq n$ and $l=j$, then for a.e. $\vec{\xi}_{n} \in \mathbb{R}^{n}$ (hence $P_{Y_{n}}$ a.e. $\vec{\xi}_{n} \in \mathbb{R}^{n}$ )

$$
\begin{aligned}
E\left[G_{Y} \mid Y_{n}\right]\left(\vec{\xi}_{n}\right)= & \alpha_{j}\left(s_{2}\right)\left(b\left(s_{1}\right)-b\left(t_{j-1}\right)\right) \\
& +\left(a\left(s_{1}\right)-[a]_{b}\left(s_{1}\right)+\left[\vec{\xi}_{n}\right]_{b}\left(s_{1}\right)\right) \\
& \times\left(a\left(s_{2}\right)-[a]_{b}\left(s_{2}\right)+\left[\vec{\xi}_{n}\right]_{b}\left(s_{2}\right)\right) .
\end{aligned}
$$

(3) If $l=n+1$ and $j \leq n$, then for a.e. $\vec{\xi}_{n}=\left(\xi_{1}, \ldots, \xi_{n}\right) \in$ $\mathbb{R}^{n}$ (hence $P_{Y_{n}}$ a.e. $\vec{\xi}_{n} \in \mathbb{R}^{n}$ )

$$
\begin{aligned}
E\left[G_{Y} \mid Y_{n}\right]\left(\vec{\xi}_{n}\right) \\
=\left(a\left(s_{1}\right)-[a]_{b}\left(s_{1}\right)+\xi_{n}+\beta_{n+1}\left(s_{1}\right)\left(a(t)-a\left(t_{n}\right)\right)\right) \\
\quad \times\left(a\left(s_{2}\right)-[a]_{b}\left(s_{2}\right)+\left[\vec{\xi}_{n}\right]_{b}\left(s_{2}\right)\right) .
\end{aligned}
$$


(4) If $l \leq n$ and $j=n+1$, then for a.e. $\vec{\xi}_{n}=\left(\xi_{1}, \ldots, \xi_{n}\right) \in$ $\mathbb{R}^{n}$ (hence $P_{Y_{n}}$ a.e. $\vec{\xi}_{n} \in \mathbb{R}^{n}$ )

$$
\begin{aligned}
E\left[G_{Y} \mid Y_{n}\right]\left(\vec{\xi}_{n}\right) \\
=\left(a\left(s_{2}\right)-[a]_{b}\left(s_{2}\right)+\xi_{n}+\beta_{n+1}\left(s_{2}\right)\left(a(t)-a\left(t_{n}\right)\right)\right) \\
\quad \times\left(a\left(s_{1}\right)-[a]_{b}\left(s_{1}\right)+\left[\vec{\xi}_{n}\right]_{b}\left(s_{1}\right)\right) .
\end{aligned}
$$

(5) If $l=n+1$ and $j=n+1$, then for a.e. $\vec{\xi}_{n}=\left(\xi_{1}, \ldots, \xi_{n}\right) \in$ $\mathbb{R}^{n}$ (hence $P_{Y_{n}}$ a.e. $\vec{\xi}_{n} \in \mathbb{R}^{n}$ )

$$
\begin{aligned}
& E\left[G_{Y} \mid Y_{n}\right]\left(\vec{\xi}_{n}\right) \\
&=\xi_{n}^{2}+\xi_{n}\left(a(t)-a\left(t_{n}\right)\right) \\
& \quad \times\left(a\left(s_{1}\right)-[a]_{b}\left(s_{1}\right)+a\left(s_{2}\right)-[a]_{b}\left(s_{2}\right)\right. \\
&\left.\quad+\beta_{n+1}\left(s_{1}\right)+\beta_{n+1}\left(s_{2}\right)\right) \\
&+\left(a\left(s_{1}\right)-[a]_{b}\left(s_{1}\right)+\beta_{n+1}\left(s_{1}\right)\left(a(t)-a\left(t_{n}\right)\right)\right) \\
& \quad \times\left(a\left(s_{2}\right)-[a]_{b}\left(s_{2}\right)+\beta_{n+1}\left(s_{2}\right)\left(a(t)-a\left(t_{n}\right)\right)\right) \\
&+\beta_{n+1}\left(s_{1}\right) \beta_{n+1}\left(s_{2}\right)\left(b(t)-b\left(t_{n}\right)\right) \\
&+ \alpha_{n+1}\left(s_{2}\right)\left(b\left(s_{1}\right)-b\left(t_{n}\right)\right) .
\end{aligned}
$$

Proof. For $\vec{\xi}_{n}=\left(\xi_{1}, \ldots, \xi_{n}\right) \in \mathbb{R}^{n}$, let $\vec{\xi}_{n+1}=\left(\xi_{1}, \ldots, \xi_{n}, \xi_{n+1}\right)$. (1) and (2) follow immediately from Theorem 23. If $s \in\left[t_{n}, t\right]$, then

$$
\begin{aligned}
& {\left[\frac{1}{2 \pi\left(b(t)-b\left(t_{n}\right)\right)}\right]^{1 / 2}} \\
& \quad \times \int_{\mathbb{R}}\left[\vec{\xi}_{n+1}\right]_{b}(s) \\
& \quad \times \exp \left\{-\frac{\left(\xi_{n+1}-\xi_{n}-\left(a(t)-a\left(t_{n}\right)\right)\right)^{2}}{2\left(b(t)-b\left(t_{n}\right)\right)}\right\} d \xi_{n+1} \\
& =\left[\frac{1}{2 \pi\left(b(t)-b\left(t_{n}\right)\right)}\right]^{1 / 2} \\
& \quad \times \int_{\mathbb{R}}\left(\xi_{n}+\beta_{n+1}(s)\left(\xi_{n+1}-\xi_{n}\right)\right) \\
& \quad \times \exp \left\{-\frac{\left(\xi_{n+1}-\xi_{n}-\left(a(t)-a\left(t_{n}\right)\right)\right)^{2}}{2\left(b(t)-b\left(t_{n}\right)\right)}\right\} d \xi_{n+1} \\
& =\xi_{n}+\beta_{n+1}(s)\left(a(t)-a\left(t_{n}\right)\right)
\end{aligned}
$$

by which (3) and (4) follow. If $s_{1}, s_{2} \in\left[t_{n}, t\right]$, then

$$
\begin{aligned}
& {\left[\frac{1}{2 \pi\left(b(t)-b\left(t_{n}\right)\right)}\right]^{1 / 2}} \\
& \quad \times \int_{\mathbb{R}}\left[\vec{\xi}_{n+1}\right]_{b}\left(s_{1}\right)\left[\vec{\xi}_{n+1}\right]_{b}\left(s_{2}\right) \\
& \quad \times \exp \left\{-\frac{\left(\xi_{n+1}-\xi_{n}-\left(a(t)-a\left(t_{n}\right)\right)\right)^{2}}{2\left(b(t)-b\left(t_{n}\right)\right)}\right\} d \xi_{n+1} \\
& =\left[\frac{1}{2 \pi\left(b(t)-b\left(t_{n}\right)\right)}\right]^{1 / 2} \\
& \quad \times \int_{\mathbb{R}}\left(\xi_{n}+\beta_{n+1}\left(s_{1}\right)\left(\xi_{n+1}-\xi_{n}\right)\right) \\
& \quad \times\left(\xi_{n}+\beta_{n+1}\left(s_{2}\right)\left(\xi_{n+1}-\xi_{n}\right)\right) \\
& \quad \times \exp \left\{-\frac{\left(\xi_{n+1}-\xi_{n}-\left(a(t)-a\left(t_{n}\right)\right)\right)^{2}}{2\left(b(t)-b\left(t_{n}\right)\right)}\right\} d \xi_{n+1} \\
& =\xi_{n}^{2}+\xi_{n}\left(a(t)-a\left(t_{n}\right)\right)\left(\beta_{n+1}\left(s_{1}\right)+\beta_{n+1}\left(s_{2}\right)\right) \\
& \quad+\beta_{n+1}\left(s_{1}\right) \beta_{n+1}\left(s_{2}\right)\left(\left(a(t)-a\left(t_{n}\right)\right)^{2}+b(t)-b\left(t_{n}\right)\right)
\end{aligned}
$$

by which we have (5).

For $j=1, \ldots, n+1$, let $h_{j}=\chi_{\left(t_{j-1}, t_{j}\right]} h$ and $g_{j}=$ $h_{j} / \sqrt{b\left(t_{j}\right)-b\left(t_{j-1}\right)}$. Let $V$ be the subspace of $L_{2}[0, t]$ generated by $\left\{g_{1}, \ldots, g_{n+1}\right\}, V^{\perp}$ the orthogonal complement of $V$, and $\mathscr{P}^{\perp}: L_{2}[0, t] \rightarrow V^{\perp}$ the orthogonal projection. Let $\mathscr{M}\left(L_{2}[0, t]\right)$ be the class of all $\mathbb{C}$-valued Borel measures of bounded variation over $L_{2}[0, t]$, and let $\mathcal{S}_{w_{\varphi_{\alpha}}}$ be the space of all functions $F$ which for $\sigma \in \mathscr{M}\left(L_{2}[0, t]\right)$ have the form

$$
F(x)=\int_{L_{2}[0, t]} \exp \{i(v, x)\} d \sigma(v)
$$

for $w_{\varphi_{\alpha}}$ a.e. $x \in C[0, t]$. Note that $\mathcal{S}_{w_{\varphi_{\alpha}}}$ is a Banach algebra [4].

Now, we have the following theorems which are our final results.

Theorem 25. Let $h$ be of bounded variation and a absolutely continuous on $[0, t]$. Let $F \in \mathcal{S}_{w_{\varphi_{\alpha}}}$ and $\sigma \in \mathscr{M}\left(L_{2}[0, t]\right)$ be related by (40). Then,

$$
E\left[F_{Y}\right]=\int_{L_{2}[0, t]} \exp \left\{-\frac{1}{2}\|v h\|^{2}\right\} d \sigma_{a}(v),
$$

where $\left(d \sigma_{a} / d \sigma\right)(v)=\exp \{i(v, a)\}$ for $v \in L_{2}[0, t]$. Moreover for a.e. $\vec{\xi}_{n+1} \in \mathbb{R}^{n+1}$ (hence for $P_{Y_{n+1}}$ a.e. $\left.\vec{\xi}_{n+1} \in \mathbb{R}^{n+1}\right) E\left[F_{Y} \mid\right.$ $\left.Y_{n+1}\right]\left(\vec{\xi}_{n+1}\right)$ is given by

$E\left[F_{Y} \mid Y_{n+1}\right]\left(\vec{\xi}_{n+1}\right)$

$=\int_{L_{2}[0, t]} \exp \left\{i\left(v, a-[a]_{b}+\left[\vec{\xi}_{n+1}\right]_{b}\right)-\frac{1}{2}\left\|\mathscr{P}^{\perp}(v h)\right\|^{2}\right\} d \sigma(v)$. 
Proof. By Corollary 5 and the Fubini's theorem

$$
\begin{aligned}
E\left[F_{Y}\right] & =\int_{L_{2}[0, t]} \int_{C[0, t]} \exp \{i(v, Y(x, \cdot))\} d w_{\varphi_{\alpha}}(x) d \sigma(v) \\
& =\int_{L_{2}[0, t]} \exp \{i(v, a)\} \exp \left\{-\frac{1}{2}\|v h\|^{2}\right\} d \sigma(v),
\end{aligned}
$$

where the last equality follows from the following integral formula:

$$
\int_{\mathbb{R}} \exp \left\{-a u^{2}+i b u\right\} d u=\left(\frac{\pi}{a}\right)^{1 / 2} \exp \left\{-\frac{b^{2}}{4 a}\right\}
$$

for $a \in \mathbb{C}_{+}$and any real $b$. Furthermore, for a.e. $\vec{\xi}_{n+1} \in \mathbb{R}^{n+1}$, we have by Theorems 3.4 and 3.6 in [8]

$$
\begin{aligned}
& E\left[F_{Y} \mid Y_{n+1}\right]\left(\vec{\xi}_{n+1}\right) \\
& =\int_{L_{2}[0, t]} \exp \left\{i\left(v, a-[a]_{b}+\left[\vec{\xi}_{n+1}\right]_{b}\right)\right\} \\
& \quad \times \int_{C[0, t]} \exp \left\{i\left(v, Z(x, \cdot)-[Z(x, \cdot)]_{b}\right)\right\} \\
& =\int_{L_{2}[0, t]} \exp \left\{i\left(v, a-[a]_{b}+\left[\vec{\xi}_{n+1}\right]_{b}\right)\right\} \\
& \quad \times \exp \left\{-\frac{1}{2}\left\|\mathscr{P}^{\perp}(v h)\right\|^{2}\right\} d \sigma(v)
\end{aligned}
$$

which completes the proof.

Theorem 26. Let the assumptions be as given in Theorem 25 and for $\vec{\xi}_{n} \in \mathbb{R}^{n}$ let

$$
A\left(v, \vec{\xi}_{n}\right)=\exp \left\{i\left(v, a-[a]_{b}+\left[\vec{\xi}_{n}\right]_{b}\right)-\frac{1}{2}\left\|\mathscr{P}^{\perp}(v h)\right\|^{2}\right\}
$$

Then, for a.e. $\vec{\xi}_{n} \in \mathbb{R}^{n}$ (hence for $P_{Y_{n}}$ a.e. $\vec{\xi}_{n} \in \mathbb{R}^{n}$ ) E[F $\left.Y_{n}\right]\left(\vec{\xi}_{n}\right)$ is given by

$$
\begin{aligned}
E\left[F_{Y} \mid Y_{n}\right]\left(\vec{\xi}_{n}\right) & \\
=\int_{L_{2}[0, t]} A\left(v, \vec{\xi}_{n}\right) \exp \{ & \left\{\frac{\left\langle v h, g_{n+1}\right\rangle\left(a(t)-a\left(t_{n}\right)\right)}{\sqrt{b(t)-b\left(t_{n}\right)}}\right. \\
& \left.-\frac{1}{2}\left\langle v h, g_{n+1}\right\rangle^{2}\right\} d \sigma(v),
\end{aligned}
$$

where $\langle\cdot, \cdot\rangle$ denotes the inner product on $L_{2}[0, t]$.
Proof. Let $\vec{\xi}_{n}=\left(\xi_{1}, \ldots, \xi_{n}\right) \in \mathbb{R}^{n}$ and let $\vec{\xi}_{n+1}=\left(\xi_{1}, \ldots, \xi_{n}\right.$, $\left.\xi_{n+1}\right)$. By the definition of the Paley-Wiener-Zygmund integral it is not difficult to show

$$
\left(v,\left[\vec{\xi}_{n+1}\right]_{b}\right)=\left(v,\left[\vec{\xi}_{n}\right]_{b}\right)+\frac{\left\langle v h, g_{n+1}\right\rangle}{\sqrt{b(t)-b\left(t_{n}\right)}}\left(\xi_{n+1}-\xi_{n}\right)
$$

so that we have for a.e. $\vec{\xi}_{n}=\left(\xi_{1}, \ldots, \xi_{n}\right) \in \mathbb{R}^{n}$

$$
\begin{aligned}
& E\left[F_{Y} \mid Y_{n}\right]\left(\vec{\xi}_{n}\right) \\
& =\left[\frac{1}{2 \pi\left(b(t)-b\left(t_{n}\right)\right)}\right]^{1 / 2} \\
& \times \int_{L_{2}[0, t]} A\left(v, \vec{\xi}_{n}\right) \\
& \times \int_{\mathbb{R}} \exp \left\{i \frac{\left\langle v h, g_{n+1}\right\rangle}{\sqrt{b(t)-b\left(t_{n}\right)}}\left(\xi_{n+1}-\xi_{n}\right)\right. \\
& \left.-\frac{\left(\xi_{n+1}-\xi_{n}-\left(a(t)-a\left(t_{n}\right)\right)\right)^{2}}{2\left(b(t)-b\left(t_{n}\right)\right)}\right\} d \xi_{n+1} d \sigma(v) \\
& =\int_{L_{2}[0, t]} A\left(v, \vec{\xi}_{n}\right) \exp \left\{i \frac{\left\langle v h, g_{n+1}\right\rangle\left(a(t)-a\left(t_{n}\right)\right)}{\sqrt{b(t)-b\left(t_{n}\right)}}\right. \\
& \left.-\frac{1}{2}\left\langle v h, g_{n+1}\right\rangle^{2}\right\} d \sigma(v)
\end{aligned}
$$

by Theorem 25 and the change of variable theorem.

\section{Acknowledgment}

This research was supported by Basic Science Research Program through the National Research Foundation (NRF) of Korea funded by the Ministry of Education, Science and Technology (2012-0002477).

\section{References}

[1] J. Yeh, "Inversion of conditional expectations," Pacific Journal of Mathematics, vol. 52, pp. 631-640, 1974.

[2] C. Park and D. Skoug, "A simple formula for conditional Wiener integrals with applications," Pacific Journal of Mathematics, vol. 135, no. 2, pp. 381-394, 1988.

[3] S. J. Chang and D. Skoug, "The effect of drift on conditional Fourier-Feynman transforms and conditional convolution products," International Journal of Applied Mathematics, vol. 2, no. 4, pp. 505-527, 2000.

[4] M. K. Im and K. S. Ryu, "An analogue of Wiener measure and its applications," Journal of the Korean Mathematical Society, vol. 39, no. 5, pp. 801-819, 2002. 
[5] D. H. Cho, "A simple formula for an analogue of conditional Wiener integrals and its applications," Transactions of the American Mathematical Society, vol. 360, no. 7, pp. 3795-3811, 2008.

[6] D. H. Cho, "A simple formula for an analogue of conditional Wiener integrals and its applications. II," Czechoslovak Mathematical Journal, vol. 59, no. 2, pp. 431-452, 2009.

[7] S. J. Chang and D. M. Chung, "Conditional function space integrals with applications," The Rocky Mountain Journal of Mathematics, vol. 26, no. 1, pp. 37-62, 1996.

[8] D. H. Cho, "A simple formula for a generalized conditional Wiener integral and its applications," International Journal of Mathematical Analysis, vol. 7, no. 29-32, pp. 1419-1431, 2013.

[9] R. B. Ash, Real Analysis and Probability, Academic Press, New York, NY, USA, 1972.

[10] R. H. Cameron and W. T. Martin, "Transformations of Wiener integrals under translations," Annals of Mathematics, vol. 45, pp. 386-396, 1944.

[11] C. Park and D. Skoug, "A Kac-Feynman integral equation for conditional Wiener integrals," Journal of Integral Equations and Applications, vol. 3, no. 3, pp. 411-427, 1991. 


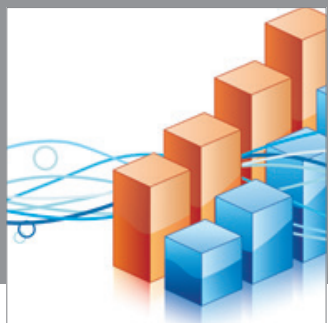

Advances in

Operations Research

mansans

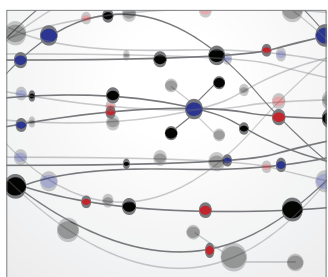

The Scientific World Journal
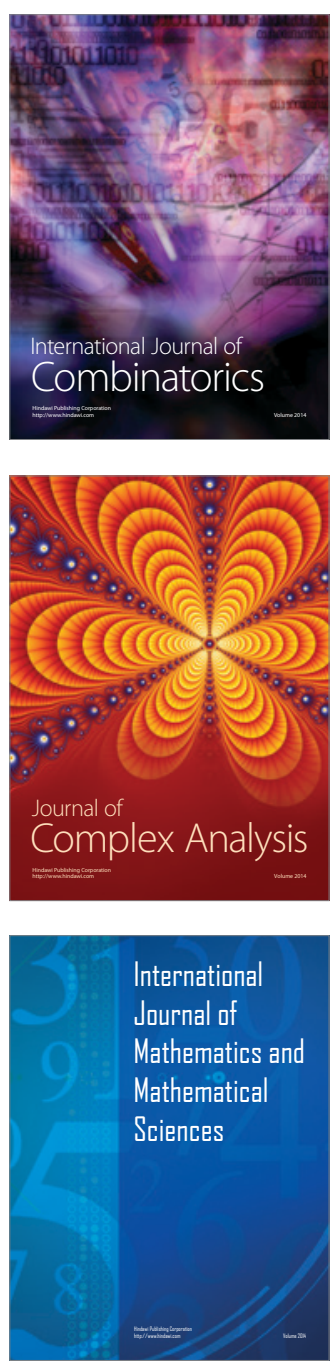
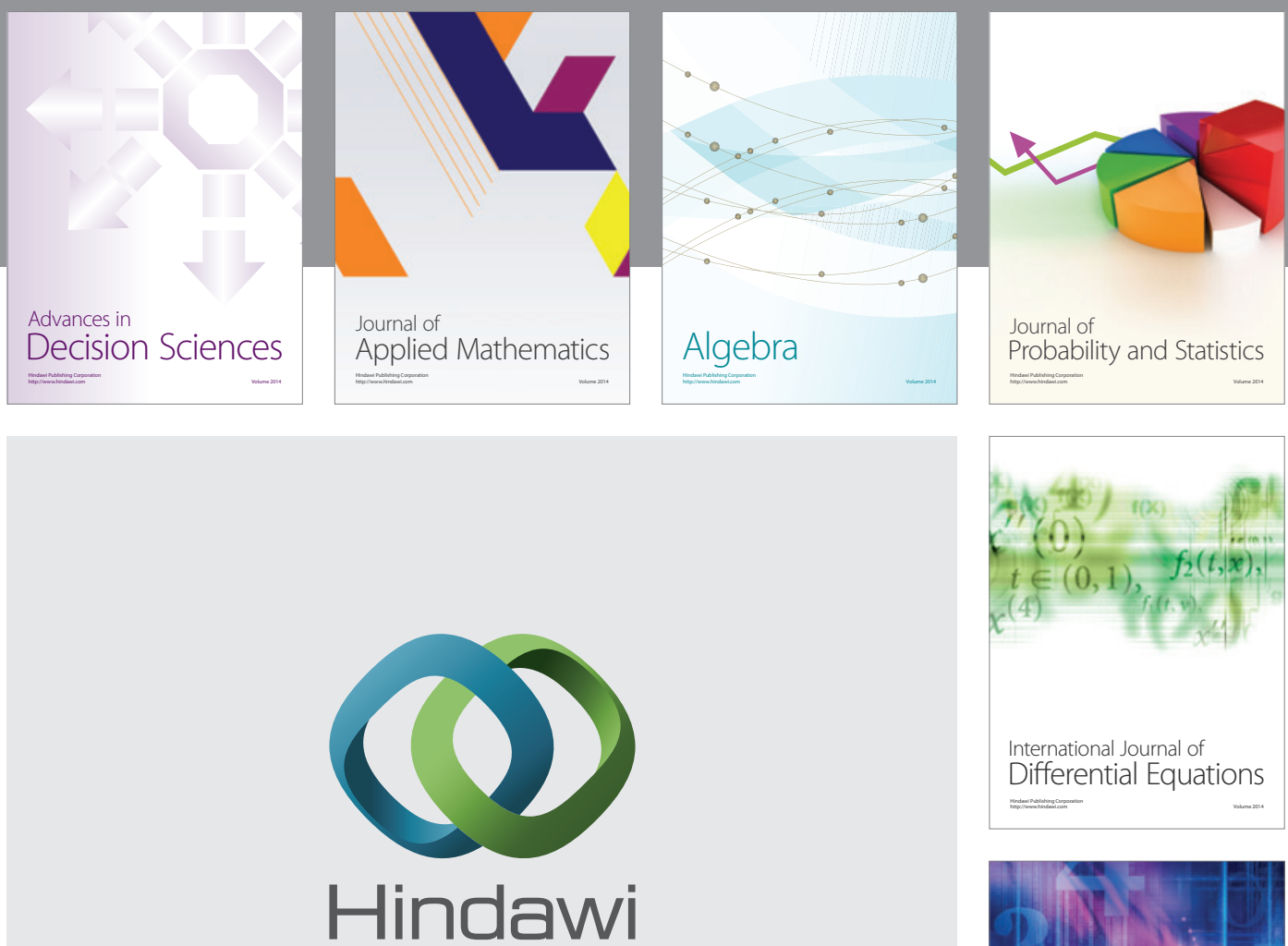

Submit your manuscripts at http://www.hindawi.com
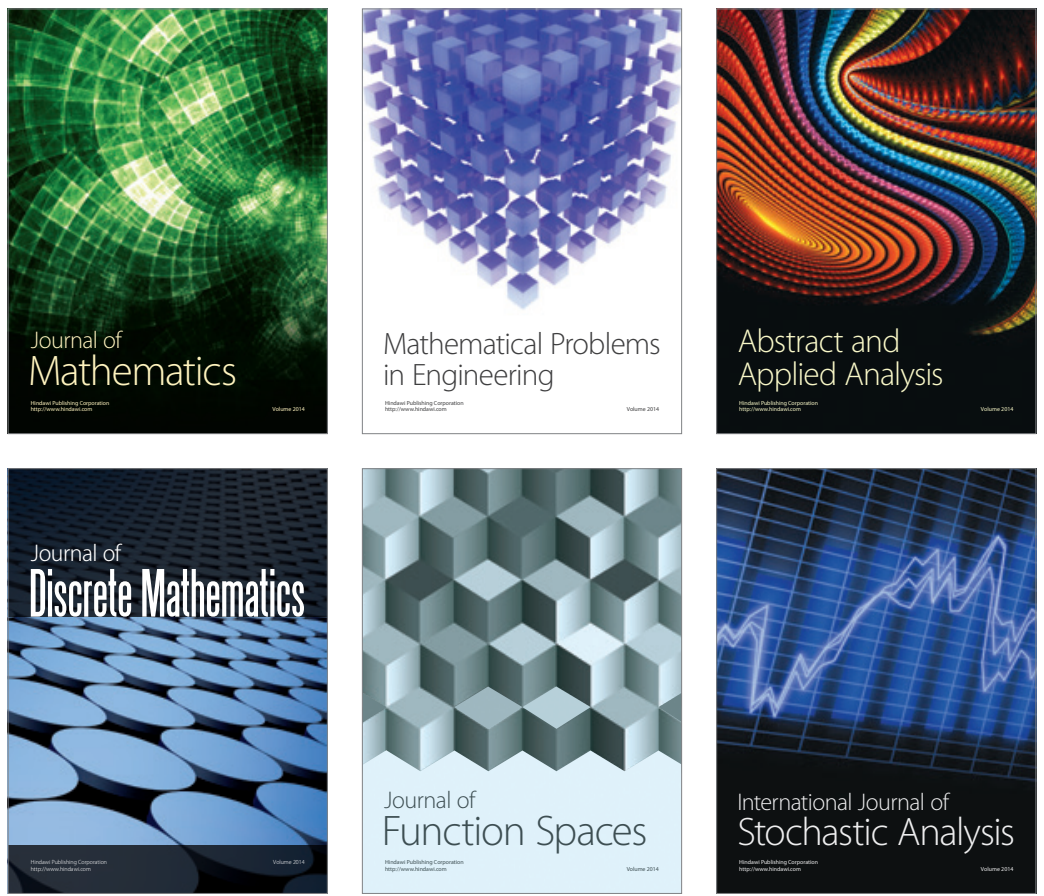

Journal of

Function Spaces

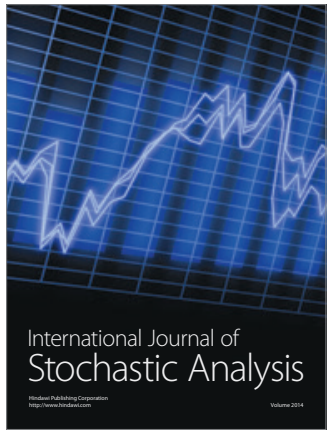

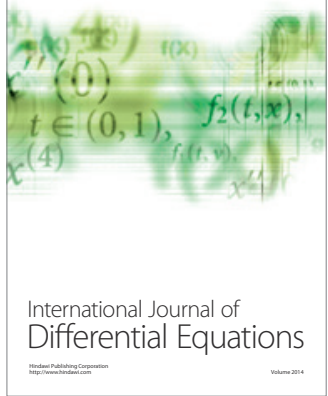
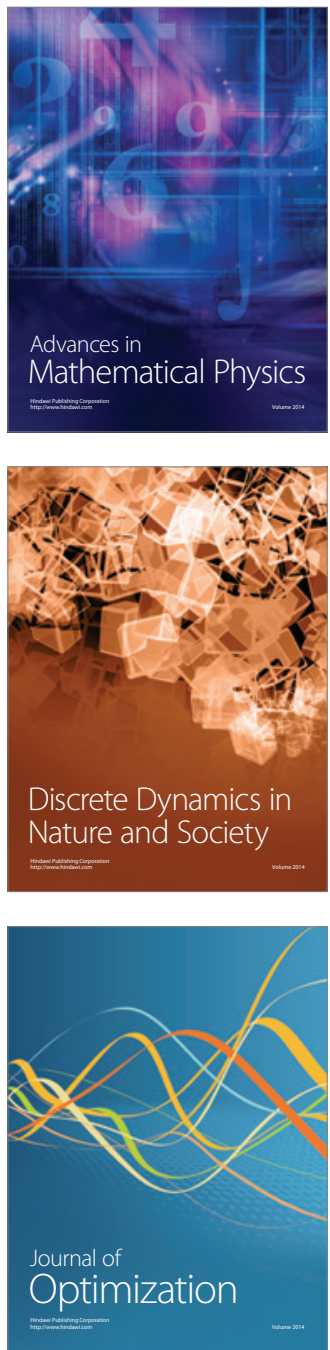